\title{
Analyzing the Diaolou's Construction and Eco-design of Qiang Minority Hong Zhang ${ }^{1,2, a}$, Xiao Hong Wang ${ }^{2}$ \\ ${ }^{1}$ School of History and Culture, Sichuan University, Chengdu, China. \\ ${ }^{2}$ Associate professor, Chengdu University, Chengdu, China \\ a E-mail: 10071983@qq.com ,Tel: +86+13388185305
}

Keywords: Diaolou; construction; housing eco-design; artistic value

\begin{abstract}
Taking the Heihu Diaolou of the Qiang Minority in the upper reaches of Mingjiang River as an example, the author analyzes the distributing, construction, functions, sorts and scales of the Qiang Diaolou, trying to reveal us the special housing eco-design feature consisted in the Diaolou.
\end{abstract}

\section{Introduction}

The ecological architecture refers to the organic combination of buildings and natural environment, which is based on the local natural environment and to reasonably arrange and organize the relationship of the buildings and the other relevant factors under the theory of ecology and architectural technology and science and modern scientific technology and means. Figure 1 show it should have the favorable indoor climatic condition and stronger ability of regulating bio-climate to satisfy the people's comfortable living environment and establish a benign circulatory system between human, buildings and natural eco-environment.

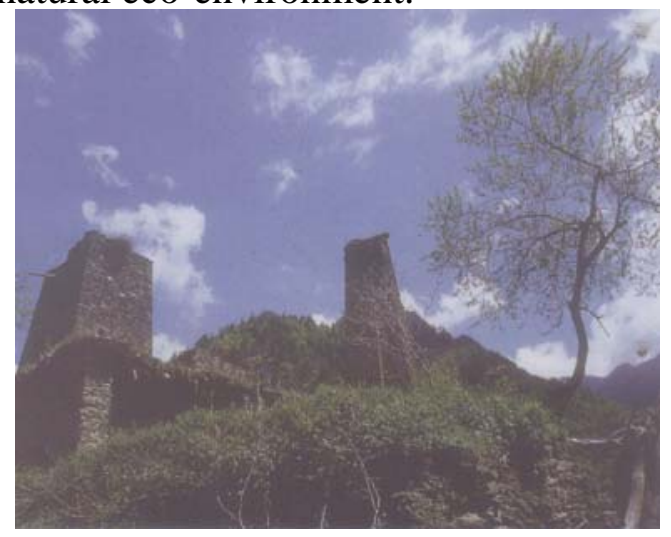

Figure. 1 Qiang's Diaolou built on the mountain

The Qiang's Diaolou in Sichuan Province is typical of the local architecture, which are full of local characteristics, national styles and ecological characteristics.

The Qiang's Diaolou shows its characteristics in site selection, construction material, space combination, structure treatment, architecture design and other aspects. In the process of construction, it should actively create a good living environment in the most ingenious and economic way based on protection of the natural environment, to pursue harmonious coexistence between human and nature [1].

\section{Site selection}

\section{Feng Shui culture}

A book named "Yuanzhi" written by Jicheng, the famous gardener in Ming Dynasty, had emphasized the importance "Xiang Di" (means refined selection of a site) in the first chapter, and analyzed the features of terrain and environment in details, thus pointed out how to utilize different kind of terrain conditions and what results may be achieved [2].

Qiang's village is Located in a high mountain valley, surrounded by Snow Mountains at the back and hills on the left and right, with sufficient spring in the front. It is consistent with the layout of 
traditional dwelling and the geomancy principle "Fuyin Baoyang".

According to the analysis of modern ecology, it is an excellent living environment. Mountains at the back can prevent the wind in winter; Facing the water, the people can enjoy the wet breeze from the southeast in summer and be provided water safeguard for producing and living; Open front, there are good sunshine and ventilated condition; The surrounding hills can prevent wind and flood, to form a micro-climate with good ecological cycle, as shown in the Figure 2.

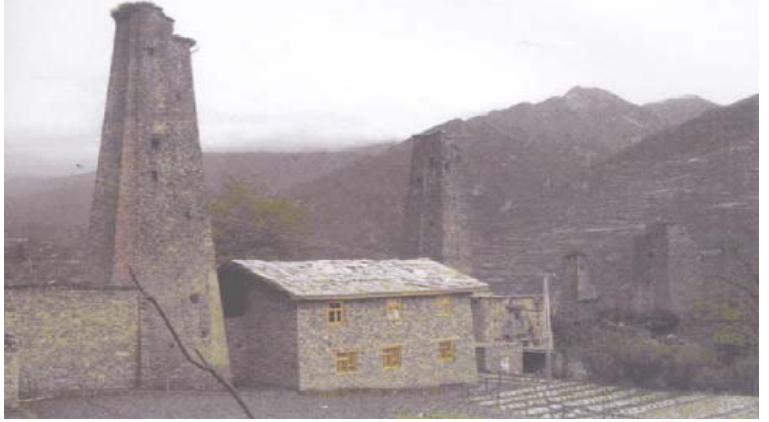

Figure. 2 Well-preserved Qiang's Diaolou

\section{Spatial rhythms}

It is widely popular that the rhythmic beauty embodied in the architecture. Both Western or Chinese building, and either ancient or modern building, around which, we can clearly feel a sense of rhythm and cadence of space. So architecture is called the solidification of music according to Goethe [3].

The rhythm and cadence of space embodied in Qiang's Diaolou are mainly in the following aspects:

1) It embodied in the overall visual effect of the building group. Because the Diaolou mostly were built near the mountain and by the river.The tilt of the mountain makes the buildings' space level abundant, just like "pyramid". Under the shade of green leaves, the yellow and white outside wall of the Diaolou was dotted interesting and orderly.

2) It embodied in the visual effect of each small unit of space.

There are three or four floors in common Diaolou, it was divided into yard, balcony, platforms and other forms according to the different function of each floor, to form the contrast between large and small, emptiness and fullness, Lights and Shadows, but the unity of various parts make the building harmony as a whole, and the rhythm of space is naturally emerged. Not to mention the Diaolou with dozen floors.

3) Because there are different terrains like flat lands and hills, it will form the contrast in the location, in the density of buildings' distribution, in the sizes of the buildings, in visual effects and other spatial forms, the changes produced aesthetic displacement, and then the rhythm was formed.

\section{Building Materials}

The major building materials of Heihu Diaolou and all the other Diaolous are: (1) stones ;(2) yellow mud; (3) wood.

All the Qiang's Diaolou which are made of harmless natural materials, reflect their own characteristics, pursue the harmony with the nature.

Stone and yellow mud can absorb a lot of heat during the day and store it, and release them slowly in the evening, making the room warm in winter, cool in summer.

This is the common way to regulate the micro-climate in traditional dwellings, make full use of natural resources, and also ensure the comfort of human habitation.

Some inherent properties of the stone, such as quality, hardness, refractory and moisture resistant, make it play a greater role in housing construction. The Diaolou is made of stone which can be obtained locally. The application in the Diaolou's construction mainly embodied its hardness, fire resistance, anti-corrosion and decay and other natural features. Because of its hardness and practical features, it is widely used in building the walls, window's platform, pillar, thresholds, steps, etc. 
One of the most powerful properties of yellow mud is plasticity, particularly in the southwest Sichuan, because of the special ingredients, the yellow mud has strong adhesive function, and stick to the stone to mix into the block, thus become the body of Diaolou's construction [4].

The wood in the Diaolou has a noise, sound-absorbing effect, which is beneficial to human health; The wood, with strong penetrability, can absorb radiation; while other general building materials can not absorb radiation which is bad for human health; The wood can maintain the good natural environment, with moisture absorption function. The cell pores in the internal structure of the wood can absorb excess moisture in the air for conditioning indoor humidity; the wood also has the function of earthquake resistance, play a role of buffering and deceleration the movement of the crust; In addition, it also has diffusivity, insulation and other properties. We could learn from the Figure 3.

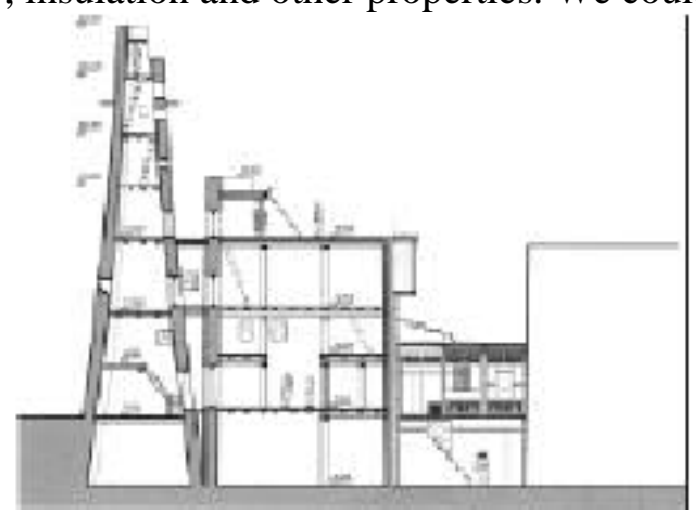

Figure. 3 Qiang's Diaolou in section

\section{Structure}

\section{Structural energy-saving}

First, the roof terrace. The bottom of the platform is used by wood or stone which are covered by the branches of bamboo and tree tightly, and then mixed with the soils and chicken manure compacted by hammer. Making the joint between the floor and wall not leak rain and snow, while ensuring the indoor warm in winter, cool in summer.

Second, opening the window. The Qiang people live in the alpine mountain areas with strong wind in four seasons. The doors and windows of the house open facing the south or southeast to keep warm, they are not open in the north, east and west walls and the bottom of the building. Figure 4 show the window facing the south is wedge-shaped with different sizes, bigger inside and smaller outside. It has keeping against cold, ventilation, heat insulation, day-lighting, security, defense and other multiple functions.

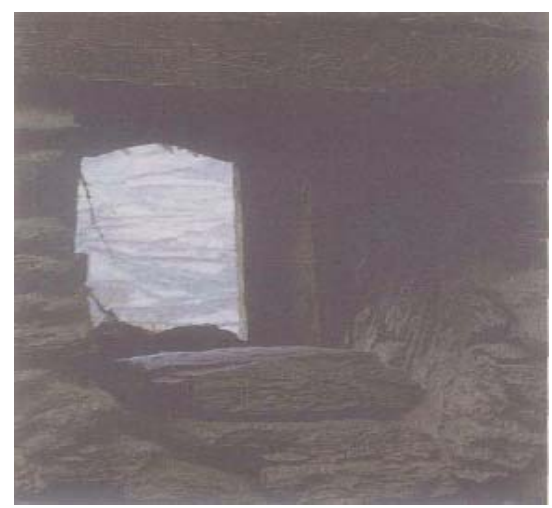

Figure. 4 Diaolou's window

Third, the ground is made of stone paving boards. It is called "permeable ground" which is different from the hardened ground. Hardened ground is used of concrete or brick paving stones, the surface is dense and impermeable, which brings a lot of harm to the living environment: (1)it may affect the flood discharge. When it rains, it prevent the infiltration of rainwater, so Roads will be covered with accumulated water after rain. When it is sunny, the ground will be extremely dry and dusty. (2)it will reflect, retain, release a lot of sun's radiation, increase the urban heat island effect, 
greatly reduce the environmental amenity; (3)it also block up the way of the rainwater directly recharge groundwater, affect the growth of vegetation, aggravating the trend of drought and water scarcity. The permeable ground is just the opposite, it can promote the infiltration of rainwater into the ground, making the ground cool in summer, warm in winter, so it greatly improve the comfort of living environment, with good ecological results. [4]

\section{The sense of layers and visual changes}

Qiang's Diaolou characterized by simple geometric designs. How to process the relation between virtuality and reality, concave and convex of walls will directly influence the spatial organization of building. The contrast between virtuality and reality, concave and convex existed in Diaolou's construction seem to be superficially opposite but actually complementary. In the interior construction spacing, there are some structural designs applied "virtual space" approach like windows, through which the sight can reach the internal Diaolou, making the people feel light, exquisite and graceful. And other real structural designs like walls, pillars, columns, etc. They are necessary components for structural support through which make people feel the sense of force. In architectural form and facade processing, the application of contrast between virtuality and actuality is indispensable. No reality the whole building will make the people feel weak and empty; no virtuality will make one feel dull and boring. In Diaolou's construction, they are combined together and compared each other, so that the appearance of the Diaolou looks light, exquisite, strong and forceful. The design of Diaolou's doors, windows, roof terrace, corridors, and other structures followed the rule of beauty in form and embodied the characteristics of architectural form[3].

\section{Conclusions}

In common parlance, that is "adjusting measures to local conditions", it is developed for long term in certain circumstances, which is shaped by the local climate and environment. Its contribution is increasingly significant as people currently expect to return nature. Nowadays, with the situation of energy shortage becoming more and more serious, the green building is the development tendency and the direction of the world construction, especially in China. so these traditional buildings should be studied and summarized, then put forward the systemic protection measures from thought ,action and institution or policies. The Qiang's Diaolou can provide some for the architectural design, construction energy conservation, and other human settlements' development in modern society to help and to use for reference.

\section{Acknowledgements}

Hong Zhang, 1974.12., PhD Candidate in Sichuan University. Associate professor in Chengdu University, Chengdu, China.

The work belongs to "Local culture resource protection and development project" sponsored by the humanity and social science research base of the education department of Sichuan province.

\section{References}

[1] Fuzheng Ji. Qiang Nationality's building in China, first edition. Chengdu: Southwest Jiaotong University Press, 2010, 1.

[2] Liuding Chen. The Art of Water Environment, first edition, Chongqing: Chongqing University Press,2009,12.

[3] Yigang Peng. Study on the architectural space combination, first edition, Beijing: China construction industry press, 2008,10.

[4] Xianglin Guan. Qiang Zu Diao Lou Qi Jing, Nationalities Forum, 2010,(3).pp34-35. 